\title{
Whole-heart 4D flow cardiac magnetic resonance in healthy dogs
}

Pfammatter, Nadia ; Campagna, Ivo ; Baron Toaldo, M ; Bruellmann, E ; Richter, Henning ; Kircher, Patrick R ; Dennler, Matthias

\begin{abstract}
In cardiac magnetic resonance imaging (CMR), accurate flow measurements rely on perpendicular plane-alignment with flow direction. For 2D phase contrast (PC) cardiac magnetic resonance measurements, planes have to be defined during the examination of the heart, which is time consuming and error-prone. Collection of flow information of the entire volume of the heart by a $4 \mathrm{D}$ flow CMR postpones plane alignment to postprocessing. Sampling of such a large amount of data requires acceleration of data acquisition with techniques such as SENSitivity Encoding (k-t SENSE) or Broad-use Linear Acquisition Speed-up Technique (k-t BLAST). Objectives of the study were to compare 4D flow CMR, accelerated with two different acceleration methods with the established 2D PC CMR based on assessment of stroke volume at all four cardiac valves. The values of stroke volume acquired with the 4D flow CMR SENSE did not differ significantly when compared to the 2D PC CMR SENSE at the left side of the heart (aortic and mitral valve). Significant differences between the techniques were seen at the pulmonic and tricuspid valves. Acceleration with k-t BLAST revealed significantly lower values of stroke volume at all cardiac valves, except at the mitral valve.
\end{abstract}

DOI: https://doi.org/10.17236/sat00159

Other titles: 4D Flussmessungen im Herzen mit kardialer Magnetresonanztomographie bei gesunden Hunden

Posted at the Zurich Open Repository and Archive, University of Zurich

ZORA URL: https://doi.org/10.5167/uzh-152552

Journal Article

Published Version

Originally published at:

Pfammatter, Nadia; Campagna, Ivo; Baron Toaldo, M; Bruellmann, E; Richter, Henning; Kircher, Patrick R; Dennler, Matthias (2018). Whole-heart 4D flow cardiac magnetic resonance in healthy dogs. Schweizer Archiv für Tierheilkunde, 160(5):295-303.

DOI: https://doi.org/10.17236/sat00159 


\title{
Whole-heart 4D flow cardiac magnetic resonance in healthy dogs
}

\author{
N.S. Pfammatter ${ }^{1}$, I. U. Campagna ${ }^{2}$, M. Baron Toaldo ${ }^{3}$, E. Bruellmann ${ }^{4}$, \\ H. Richter ${ }^{1}$, P. R. Kircher ${ }^{1}$, M. Dennler ${ }^{1}$ \\ ${ }^{1}$ Clinic of Diagnostic Imaging, ${ }^{2}$ Section of Anaesthesiology and ${ }^{3}$ Division of Cardiology, Vetsuisse Faculty,
} University of Zurich, Switzerland, 4 Philips AG, Zurich, Switzerland

Dr. Baron Toaldo's present address is Department of Veterinary Medical Sciences, Alma Mater Studiorum, University of Bologna, Ozzano Emilia, Italy.

\begin{abstract}
In cardiac magnetic resonance imaging (CMR), accurate flow measurements rely on perpendicular plane-alignment with flow direction. For 2D phase contrast (PC) cardiac magnetic resonance measurements, planes have to be defined during the examination of the heart, which is time consuming and error-prone. Collection of flow information of the entire volume of the heart by a $4 \mathrm{D}$ flow CMR postpones plane alignment to post-processing. Sampling of such a large amount of data requires acceleration of data acquisition with techniques such as SENSitivity Encoding ( $\mathrm{k}$-t SENSE) or Broad-use Linear Acquisition Speed-up Technique (k-t BLAST). Objectives of the study were to compare 4D flow CMR, accelerated with two different acceleration methods with the established 2D PC CMR based on assessment of stroke volume at all four cardiac valves. The values of stroke volume acquired with the 4D flow CMR SENSE did not differ significantly when compared to the $2 \mathrm{D}$ PC CMR SENSE at the left side of the heart (aortic and mitral valve). Significant differences between the techniques were seen at the pulmonic and tricuspid valves. Acceleration with k-t BLAST revealed significantly lower values of stroke volume at all cardiac valves, except at the mitral valve.
\end{abstract}

Keywords: cardiac flow quantification, canine, 4D magnetic resonance imaging, $\mathrm{k}$-t SENSE, $\mathrm{k}-\mathrm{t}-\mathrm{BLAST}$, phase contrast angiography

\section{D Flussmessungen im Herzen mit kardialer Magnetresonanztomogra- phie bei gesunden Hunden}

Bei der Herzuntersuchung mittels Magnetresonanztomographie (CMR) muss die Ebene für akkurate Flussmessungen senkrecht zur Blutflussrichtung definiert werden. Im 2D Phasenkontrast (PC) CMR erfolgt die Wahl der Ebene schon während der Untersuchung, was zeitaufwendig und problemanfällig ist. Mit der Messung aller Blutflussinformationen des gesamten Herzens mittels einer 4D flow CMR, verschiebt sich die Wahl der Ebenen in die Nachbearbeitung. Das Sammeln solch grosser Datenmengen erfordert eine Beschleunigung der Messtechnik wie SENSitivity Encoding (k-t SENSE) oder Broad-use Linear Acquisition Speed-up Technique (k-t BLAST). Das Ziel dieser Studie war, die 4D flow CMR, beschleunigt mit zwei verschiedenen Methoden, mit der etablierten 2D PC CMR anhand der Bestimmung des Schlagvolumens über allen vier Herzklappen zu vergleichen. Die Werte der Schlagvolumenmessung für die linke Seite des Herzens (Aorten- und Mitralklappe), durchgeführt anhand der 4D flow CMR k-t SENSE, waren nicht signifikant verschieden im Vergleich zu den Messungen mittels der 2D PC CMR k-t SENSE. An der Pulmonal- und Trikuspidalklappe zeigten sich jedoch signifikante Unterschiede zwischen diesen beiden Techniken. Die Beschleunigung durch k-t-BLAST ergaben generalisiert tiefere Werte des Schlagvolumens an allen Herzklappen, ausser der Mitralklappe.

Schlüsselwörter: Herzflussmessung, 4D Magnetresonanztomographie, k-t SENSE, k-t BLAST, Phasenkontrastangiographie https://doi.org/ $10.17236 /$ sat00159

Received: 25.09.2017 Accepted: 20.01.2018 
Whole-heart 4D flow cardiac magnetic resonance in healthy dogs

N.S. Pfammatter et al.

\section{Introduction}

Cardiac magnetic resonance imaging (CMR) gains popularity in veterinary diagnostic imaging (Vallee et al., 2004; Gilbert et al., 2010). CMR in animals is used for evaluation of cardiac morphology (Contreras et al., 2008; Baumwart et al., 2009; Drees et al., 2015), cardiac function (Hockings et al., 2003; MacDonald et al., 2005; Baumwart et al., 2009; Kim et al., 2013; Meyer et al., 2013; Sieslack et al., 2013; Sieslack et al., 2014; Drees et al., 2015; Drees et al., 2015) as well as associated diseases of the heart (MacDonald et al., 2006; MacDonald et al., 2006; Mai et al., 2010), the pericardium (Boddy et al., 2011), and the adjacent vasculature (Mai et al., 2010; Lee et al., 2017). Comparison of stroke volume based on a flow-sensitive phase-contrast sequence in different anatomical locations provides important clinical information such as cardiac output, shunt fraction, collateral flow around obstructions and flow differences in the pulmonary arteries (Gatehouse et al., 2005). A recently published study (Dennler et al., 2017) compared different methods of evaluation of stroke volume using a cine gradient echo sequence and a two-dimensional (2D) phase contrast (PC) CMR sequence in healthy dogs. The stroke volume of the left and right ventricle was measured based on morphology and compared with measurements of the stroke volume over all four cardiac valves acquired with a $2 \mathrm{D}$ PC CMR. The study showed, that evaluation based on flow quantification was more robust than using morphology to calculate the stroke volume. But flow quantification relies on perpendicular alignment of the region of interest (ROI) with flow direction (Bogaert et al., 2012). Therefore, planes in accurate position have to be defined before acquisition of the sequence, which is time consuming, error-prone and highly operator dependent (Lotz et al., 2002; Sargent et al., 2015; Dennler et al., 2017). To overcome this limitation, collection of cardiac flow information with a three-dimensional time-resolved (4D) flow CMR method including the whole heart allows plane alignment to post-processing (Brix et al., 2009; Attili et al., 2010, Stankovic et al., 2013), which allows reduction of anesthesia time as a single sequence is used to acquire all data from the whole heart and adjacent vasculature. The increasing application of $4 \mathrm{D}$ flow $\mathrm{CMR}$ in human medicine for intra- and extra-cardiac flow quantification resulted in a universal consensus statement about its use in 2015. (Dyverfeldt et al., 2015).

Sampling such a large amount of data in clinical patients requires acceleration of image acquisition with techniques such as SENSitivity encoding (k-t SENSE) or Broad-use Linear Acquisition Speed-up Technique (k-t BLAST). The acceleration with k-t SENSE works as parallel imaging technique and achieves a shortening in acquisition time by reducing lines in $\mathrm{k}$-space. The signals and sensitivities from a multi-element phase-array surface coil are combined in one image. The individual coil element information is used to unfold the aliasing of the data due to reduction of phase-encoding steps. The acceleration with k-t BLAST represents a different approach to accelerate imaging of dynamic objects. Repetitive imaging of static objects, such as the thoracic wall or the liver, produces redundant data. Focusing on the variable fractions in the image by measuring exclusively alternating data reduces false investment of scan time. The k-t BLAST technique uses two steps for acquisition of high-resolution images without aliasing. A first step generates an under-sampled image that would exhibit multiple fold-over artifacts if reconstructed conventionally. A second step, the training stage acquires an estimate of the novel, moving parts in the image and allows reconstruction of the image without aliasing (Stadlbauer et al., 2009; Attili et al., 2010; Stadlbauer et al., 2010; Carlsson et al., 2011).

Objectives of our study were to compare stroke volume at the aortic (AV), pulmonic (PV), mitral (MV) and tricuspid (TV) valves using whole-heart 4D flow CMR accelerated with $k-t$ SENSE and $k-t$ BLAST with a $2 \mathrm{D}$ PC CMR accelerated with k-t SENSE. The hypothesis was that no significant differences occur measuring stroke volume with different methods at all four cardiac valves.

\section{Animals, Material and Methods}

\section{Animals}

Seven healthy Beagle dogs were included in the study, 2 males and 5 females with a mean age of $3.1 \pm 0.2$ years and a mean body weight of $13.1 \pm 2.8 \mathrm{~kg}$. The dogs were considered healthy based on clinical examination, blood and urine analysis as well as echocardiographic examination by a cardiology resident (MBT). All procedures of the study followed the guidelines established by the Animal Welfare Act of Switzerland and the Cantonal Veterinary Office approved the study design (license number 144/2013).

\section{Anesthesia}

Each dog was premedicated with midazolam (Dormicum, Roche Pharma AG; Reinach, Switzerland; $0.1 \mathrm{mg}$ / kg, i.m.) and butorphanol (Morphasol, Dr. E. Graeub AG; Bern, Switzerland; $0.2 \mathrm{mg} / \mathrm{kg}$ i.m.) followed by placement of an intravenous catheter. General anesthesia was induced with propofol (Propofol 1\%, Fresenius Kabi AG; Oberdorf, Switzerland; 4 to $6 \mathrm{mg} / \mathrm{kg}$ i.v.) and after orotracheal intubation maintained with isoflurane (IsoFlo; Abott AG; Altishofen, Switzerland) combined in oxygen and room air (fraction of inspired oxygen $\left(\mathrm{FIO}_{2}\right)$ of 0.6-0.7). Simultaneously, the dogs received 
intravenous crystalloid Lactated Ringer's Solution (Ringer-Lactate, $5 \mathrm{ml} / \mathrm{kg} / \mathrm{h}$, Fresenius Kabi AG, Oberdorf, Switzerland). The patients obtained positive pressure ventilation (Mallard, Mallard Medical; U.K.) in a volume-controlled mode $(10-15 \mathrm{ml} / \mathrm{kg})$ with adaptation of the respiratory rate to achieve an end-tidal carbon dioxide of $40 \mathrm{mmHg}(5.3 \mathrm{kPa})$. The dogs were placed in dorsal recumbency with the head towards the gantry.

\section{Electrocardiography}

Cardiac electrical activity was monitored with a wireless vector cardiography unit (VCG) (Philips AG; Zurich, Switzerland) with four MR-safe electrodes (750 Clear Tape Electrodes, KendallTM, Anandic 110 Medical System SA; Feuerthalen, Switzerland) placed at the thoracic wall at the level of the heart. A peripheral pulse (PPU) unit (Philips AG; Zurich, Switzerland) served as monitor of mechanical cardiac performance. Respiration navigation was used in the volume acquisition sequences. The ROI for tracking diaphragmatic motion was placed over the right diaphragmatic crus, with one third of the ROI positioned in the lung and two thirds in the liver (Fig. 1.).

\section{Cardiac magnetic resonance and blood flow measurements}

Cardiac MRI was performed in a three Tesla unit (Philips Ingenia 3T with dStream body coil Solution, Philips
AG; Zurich, Switzerland). A board-certified radiologist was responsible for sequence planning and aligning stacks (MD). A standard three plane localizer and VCG-gated 2D PC CMR accelerated with k-t SENSE (TE: $2.9 \mathrm{~ms}$; TR: $4.7 \mathrm{~ms}$; SENSE: yes; slice thickness: 8 $\mathrm{mm}$; slice gap: $5 \mathrm{~mm}$; FOV: 180x180; Flip angle $10^{\circ}$;) were acquired in $\mathrm{AV}, \mathrm{PV}, \mathrm{MV}$ and TV planes, perpendicular to the flow direction. The peak velocity-encoding value (Venc) was set at $200 \mathrm{~cm} / \mathrm{s}$ for all valves.

The DICOM images of the 2D PC CMR were analyzed on an extended workstation (Extended work space Philips AG; Zurich, Switzerland) with a compatible software (Cardiac Explorer and Q-FlowAnalysis Philips AG; Zurich, Switzerland). A board certified veterinary radiologist (MD) analyzed the 2D PC CMR studies by outlining the region of interest, such as the vascular lumen at the valve region manually and the respective blood flow was calculated automatically.

Two different PPU-gated 4D flow CMR (TE: $1.4 \mathrm{~ms}$; TR: $4.6 \mathrm{~ms}$; slice thickness: $1.5 \mathrm{~mm}$; slice gap: $0.8 \mathrm{~mm}$; FOV: 200x220; Flip angle $20^{\circ}$ ) were obtained. Acceleration was performed once by k-t SENSE (acceleration factor of 3 ) and once by k-t BLAST (6-fold acceleration). Retrospective gating divided the cardiac cycle in 24 phases.
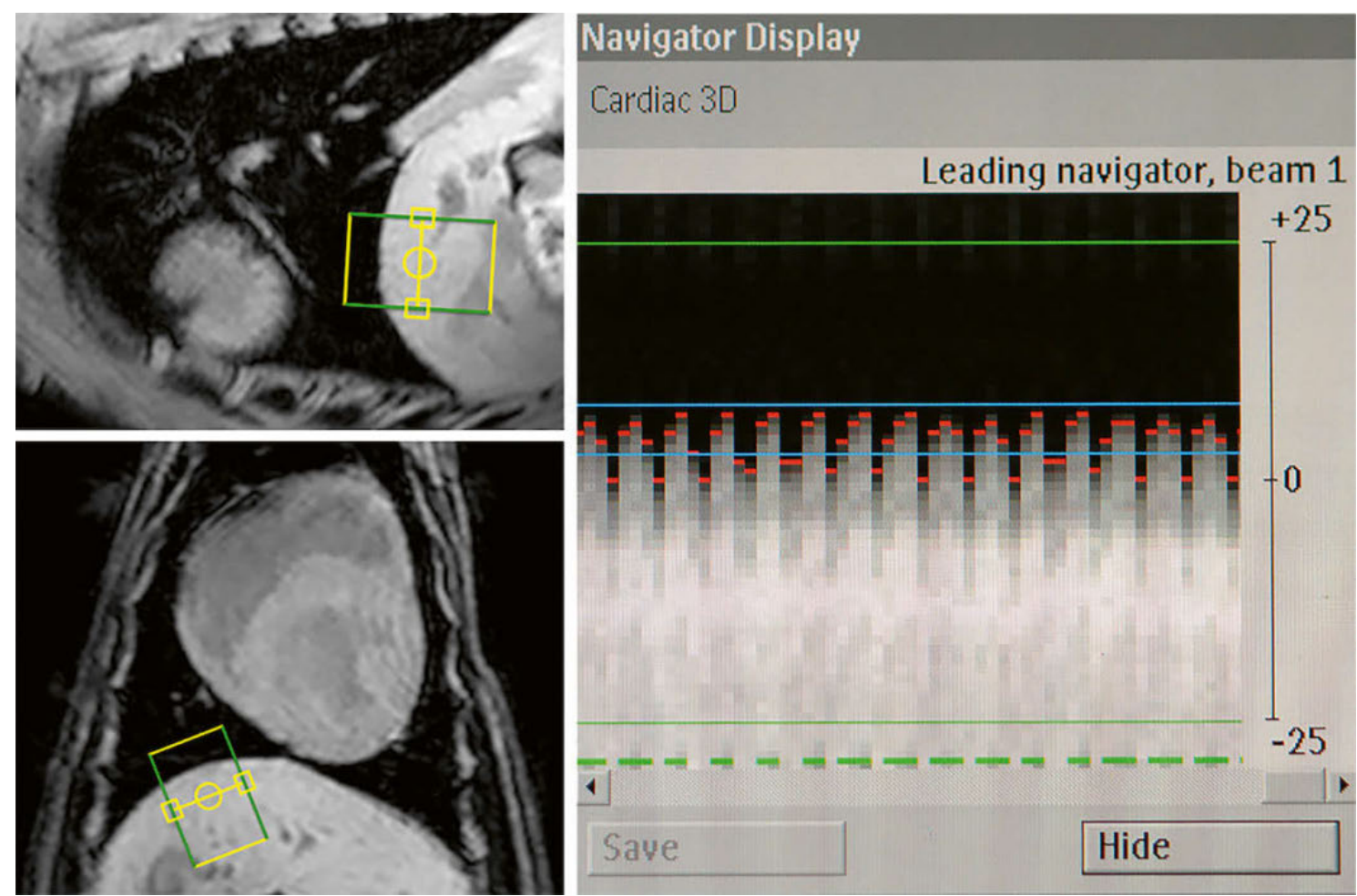

Figure 1: Respiration navigation. Respiration navigation was used in the 4D flow CMR. The ROI for tracking diaphragmatic position was placed over the right diaphragmatic crus, with one third of the ROI located in the thorax and two thirds in the abdomen. Rejection of measurements, acquired during diaphragmatic displacement, eliminates respiratory motion artifacts. 
Whole-heart 4D flow cardiac magnetic resonance in healthy dogs

N.S. Pfammatter et al.
The cine images (magnitude and velocity images) of both accelerated sequences were implemented as a separate module in a commercially available GTFlow software (GyroTools LLC, Zurich, Switzerland) and analyzed by a resident of veterinary diagnostic imaging (NSP). (Fig. 2 and Fig. 3)

The measurement plane was set perpendicular to the flow direction at $\mathrm{AV}$ and $\mathrm{PV}$ and the atrioventricular valves and a ROI was manually drawn in the velocity-encoded maps outlining the lumen of the vessel in all 24 segments of the cardiac cycle. The software generated automatically a time velocity curve shown as graph and calculated the stroke volume at the specific localization.

\section{Statistical analysis}

The statistical analyses were performed by one of the co-authors (HR) using SPSS (IBM SPSS Statistics for Windows, version 21.0, IBM Corp. Armonk, NY, USA). Due to the small number of animals, the data were analyzed as non-parametric with a Friedman test across multiple measurements, followed by a Wilcoxon Signed Ranks Test between two repeated measurements. The level of significance was set at $\mathrm{p}<0.05$.

Table 1: Mean, standard deviation, minimum and maximum values of the stroke volume measured with the 2D PC CMR accelerated with k-t SENSE and the 4D flow CMR accelerated with k-t SENSE and k-t BLAST at the four cardiac valves of seven healthy beagle dogs.

\begin{tabular}{|c|c|c|c|c|}
\hline & & $\begin{array}{c}\text { 2D k-t SENSE } \\
\text { (ml/beat) }\end{array}$ & $\begin{array}{c}\text { 4D k-t SENSE } \\
\text { (ml/beat) }\end{array}$ & $\begin{array}{c}\text { 4D k-t BLAST } \\
\text { (ml/beat) }\end{array}$ \\
\hline \multirow[t]{3}{*}{ Aortic valve } & Mean \pm StdDev & $15.59 \pm 3.56$ & $15.43 \pm 5.05$ & $8.57 \pm 1.91$ \\
\hline & Min & 12.5 & 11.1 & 5.6 \\
\hline & Max & 22.7 & 25.1 & 10.8 \\
\hline \multirow[t]{3}{*}{ Pulmonic valve } & Mean \pm StdDev & $16.01 \pm 3.40$ & $13.06 \pm 2.93$ & $7.61 \pm 1.67$ \\
\hline & Min & 11.8 & 9.9 & 4.8 \\
\hline & Max & 22.8 & 18.9 & 10.1 \\
\hline \multirow[t]{3}{*}{ Mitral valve } & Mean \pm StdDev & $15.71 \pm 4.17$ & $14.56 \pm 4.57$ & $11.29 \pm 3.01$ \\
\hline & Min & 11.8 & 10.3 & 5.6 \\
\hline & Max & 23.6 & 21.9 & 15.1 \\
\hline \multirow[t]{3}{*}{ Tricuspid valve } & Mean \pm StdDev & $15.21 \pm 2.89$ & $11.53 \pm 3.61$ & $8.57 \pm 4.04$ \\
\hline & Min & 11 & 6.4 & 1.2 \\
\hline & Max & 18.4 & 17.8 & 12.9 \\
\hline
\end{tabular}
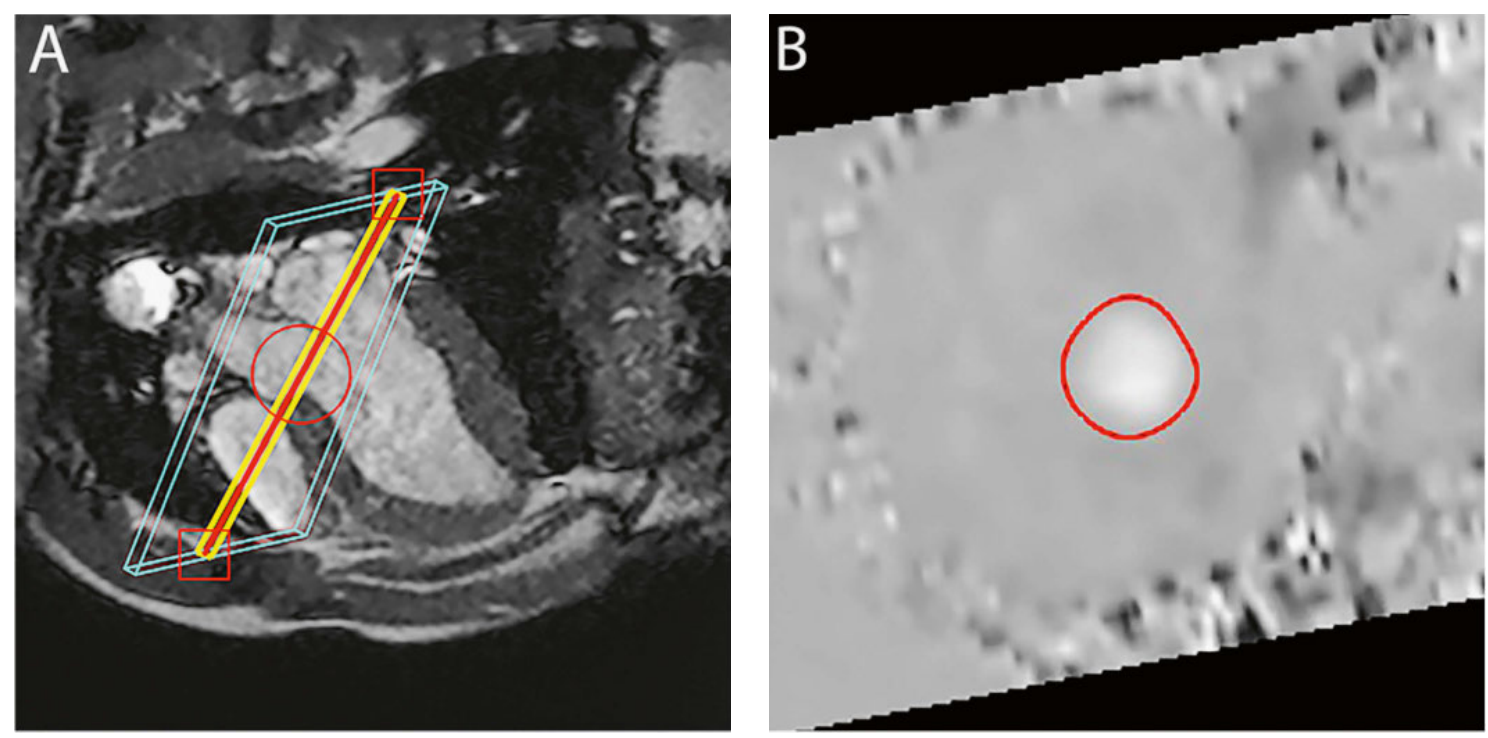

Figure 2: A: Sagittal plane of a canine heart using a time-resolved phase contrast sequence, B: associated velocity-encoding map outlining the aortic volume at the level of the aortic valves.

The stack is positioned with a short distance to the aortic valve (AV) using the sagittal plane. The ROI is manually drawn on the velocity-encoding map outlining the vascular lumen. The software (GyroTools LLC, Zurich, Switzerland) expressed the stroke volume automatically. 


\section{Results}

The seven Beagle dogs were considered healthy based on normal cardiac function and cardiac morphology examined by echocardiography and blood values within normal ranges. During the MRI examination, the systolic and diastolic blood pressure (BP) as well as the heart rate were always within physiologic ranges (BP: MAP > 60 mmHg, HR: $70-120$ beats per minute).

Stroke volume assessed using the 2D PC CMR acquired under breath-hold for $\mathrm{AV}, \mathrm{PV}, \mathrm{TV}$ and $\mathrm{MV}$ revealed $15.59 \pm 3.56 \mathrm{ml}, 16.01 \pm 3.40 \mathrm{ml}, 15.21 \pm 2.89 \mathrm{ml}$ and $15.71 \pm 4.17 \mathrm{ml}$. The 4D flow CMR k-t SENSE with respiration navigation resulted in stroke volume of 15.43
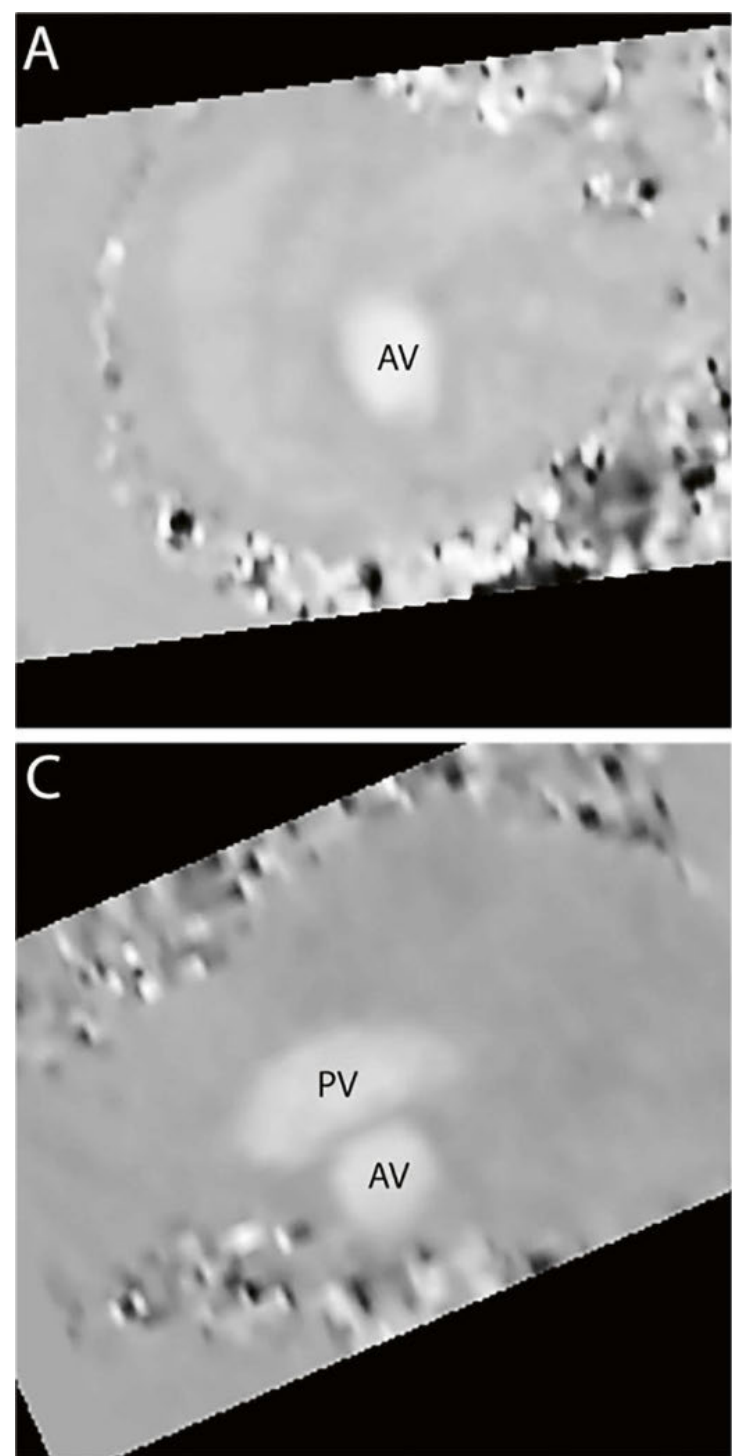

Figure 3: Velocity-encoding maps (cm/s) acquired by a 4D flow CMR. A: aortic valve (AV), B: mitral valve (MV), C: aortic and pulmonic valve (AV and PV), D: tricuspid valve (TV).

The positive through plane flow (AV and PV) is shown as bright areas and the negative through plane flow as dark areas (MV and TV) within a grey map of static tissue (myocardium).

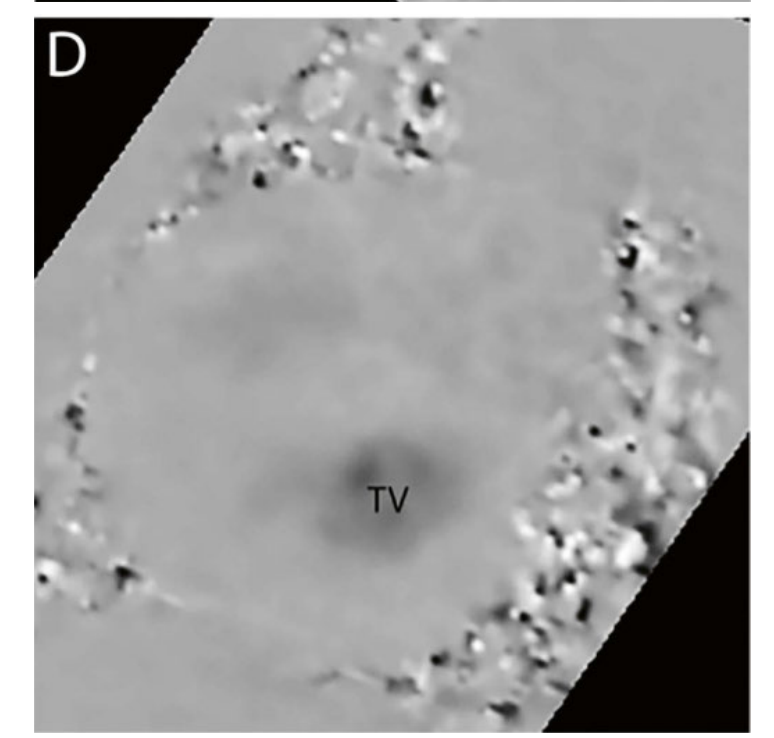

$\pm 5.05 \mathrm{ml}, 13.06 \pm 2.93 \mathrm{ml}, 11.53 \pm 3.61 \mathrm{ml}$ and 14.56 $\pm 4.57 \mathrm{ml}$ at $\mathrm{AV}, \mathrm{PV}, \mathrm{TV}$ and $\mathrm{MV}$ and the 4D flow CMR accelerated with $\mathrm{k}-\mathrm{t}$ BLAST at the same locations 8.57 $\pm 1.91 \mathrm{ml}, 7.61 \pm 1.67 \mathrm{ml}, 8.57 \pm 4.04 \mathrm{ml}$ and $11.29 \pm$ $3.01 \mathrm{ml}$. The results measured using the three different methods are summarized in Table 1.

A comparison of measurements across multiple acquisition and acceleration techniques revealed significant differences of stroke volume at 3 localizations determined by Friedman Test (AV: $p=0.004$, PV: $p=0.002$ and TV: $p=0.001)$. No significant differences occurred by Friedman Test at MV $(p=0.156)$. The observed differences at AV, PV and TV were further analyzed and confirmed using the Wilcoxon Signed Rank Test. AV

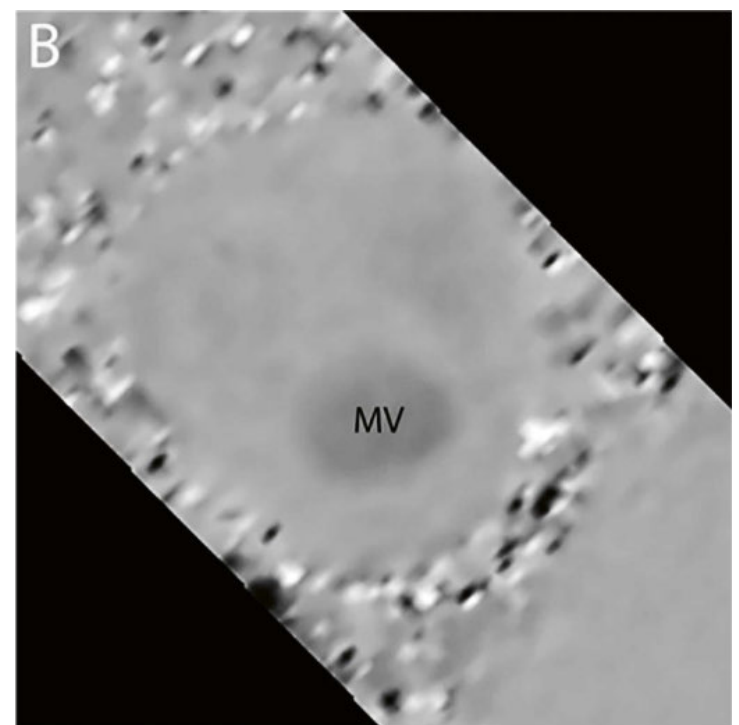

Whole-heart 4D flow cardiac magnetic resonance in healthy dogs

N.S. Pfammatter et al. 
Whole-heart 4D flow cardiac magnetic resonance in healthy dogs

N.S. Pfammatter et al. showed no significant differences comparing the 2D PC CMR k-t SENSE sequence and the 4D flow CMR k-t SENSE $(\mathrm{p}=0.31)$. Differences were significant when comparing the 2D PC CMR k-t SENSE and the 4D flow CMR k-t SENSE at PV $(p=0.028)$ and TV $(p=0.018)$ and comparing both methods with the 4D flow CMR k-t BLAST at AV, PV and TV ( $p=0.018$ for all localizations).

\section{Discussion}

The 4D flow CMR was introduced in veterinary medicine to evaluate the stroke volume at all cardiac valves. To the author's knowledge, assessment of stroke volume was not yet performed by a 4D flow CMR in healthy dogs. The volume acquisition CMR of the heart was recently used in studies of Mai et al. (2010) and Lee at al. (2017) to assess cardiac morphology and to perform a contrast-enhanced angiography of the great vessels. The hypothesis that no significant differences occur between stroke volume measured with different methods in variable locations, had to be rejected partially. On the left side of the heart, MV showed no significant differences comparing all three methods. However, on the right side of the heart, measured at PV and TV, significant differences in stroke volume between all three different methods of data acquisition could be detected.

Guidelines for ROI placement within the great cardiac vessels have been published in human medicine (Lotz et al., 2002). Therefore, the ROI within the aorta was not placed exactly at the aortic valve to prevent turbulent flow caused by valve movements and the ROI to measure the pulmonic flow was set between the pulmonic valve and the pulmonic bifurcation. Setting the planes in 2D PC CMR as well as positioning the stack for in the 4D flow CMR turned out to be more challenging in the PV and TV due to their anatomic localization and bent course. If the planes using a $2 \mathrm{D}$ PC CMR are not correctly positioned the entire sequence has to be repeated, making the acquisition period more time consuming and hence resulting in prolonged anesthesia. The main advantage of the $4 \mathrm{D}$ flow CMR is to include the whole heart and the adjacent vessels within the field of view (FOV) and the desired imaging planes can be reconstructed after acquisition, reducing the necessity of repeating sequences due to mal-positioning of planes. After placement of the stack in the 4D flow CMR images, the ROI was drawn within the velocity-encoding map outlining the vascular lumen, which was easiest to perform for AV and PV due to sharp contours of the vascular lumen and less overlap with each other. The atrioventricular valves revealed a less precise area of flow and were partially superimposed by the aorta and pul- monary artery. Therefore, ROIs at all 24 segments per cardiac cycle for all cardiac valves were drawn manually to prevent flow measurement failures due to incoming flow from another vessel.

In general, the values of stroke volume measured at the four cardiac valves performed with the 4D flow CMR accelerated with k-t SENSE were mildly lower than in 2D PC CMR k-t SENSE, which is in consensus with studies published in human medicine (Carlsson et al., 2011). Furthermore, in that study no significant differences in stroke volume measured at the aorta and main pulmonary artery were found when comparing the 4D flow CMR accelerated with k-t SENSE and the 2D PC CMR k-t SENSE sequence. The values of stroke volume in this study did show a low standard deviation in all sequences and the values measured in 2D PC CMR k-t SENSE and 4D flow CMR k-t SENSE were comparable with other studies measuring stroke volume by cardiac MRI in dogs of similar size (Hockings et al., 2003; Kim et al., 2013; Meyer et al., 2013; Sieslack et al., 2013; Sieslack et al., 2014; Drees et al., 2015; Drees et al., 2015).

The acceleration with k-t BLAST showed the lowest values of stroke volume in comparison with the methods accelerated by k-t SENSE. This is in consensus with previous studies applied in human medicine, which could show temporal blurring with k-t BLAST acceleration for stroke volume (Stadlbauer et al., 2009; Stadlbauer et al., 2010; Carlsson et al., 2011; van Ooij et al., 2013). This effect can be explained, as stroke volume is the sum of flow volumes over all heart phases. Other flow measurements, which are averaged over time, are not sensitive to temporal blurring, such as e.g. mean velocity. In human studies, additional acceleration methods were used and in a recent study EPI (echo-planar-imaging) was shown to be more stable for assessment of intra-cardiac flow measurements than k-t-SENSE and k-t-BLAST (Garg et al., 2017). Brix et al. compared the conventional 2D PC CMR with a 4D flow CMR accelerated with EPI (echo-planar-imaging) at the ascending aorta and the main pulmonary artery and no significant differences were seen. To prevent prolonging the time the animals are under anesthesia further measurements with additional acceleration techniques were avoided in this study.

An additional advantage of the 4D flow CMR was the independence of breath-hold technique. The animals could breath continuously, in our study under controlled ventilation, with a $\mathrm{CO} 2$ level in a physiological range. The dogs in our study obtained positive pressure ventilation with adaptation of the respiratory rate to achieve a physiologic end-tidal carbon dioxide (PE'CO2) of $40 \mathrm{mmHg}(5.3 \mathrm{kPa})$, which was kept constant during acquisition with respiration navigation. Respiration nav- 


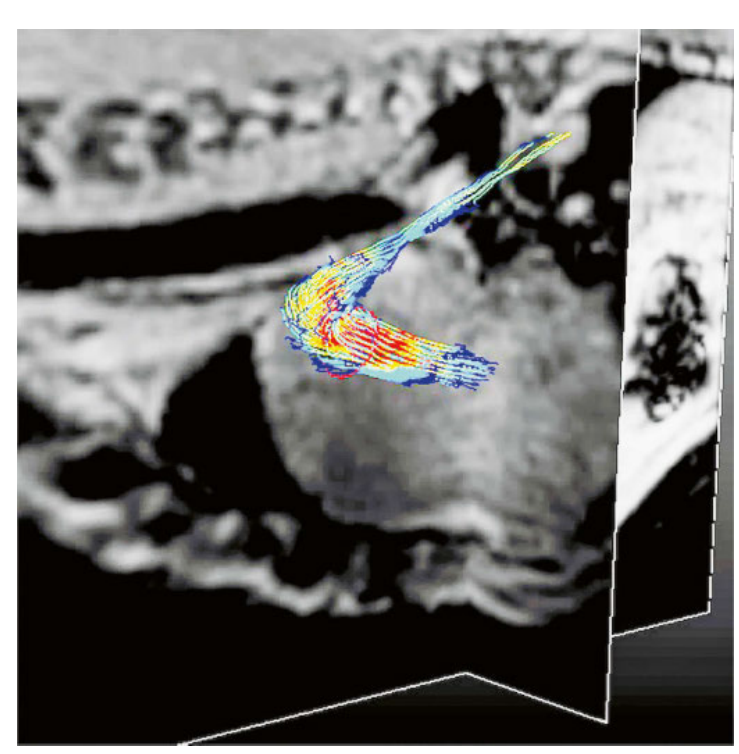

Figure 4: 3D image of the heart performed by a 4D flow CMR presenting flow through the aortic valve, plotted as streamlines.

igation is an advantage for clinical patients, especially if they have cardiac function deficits.

For breath-hold, applied during the acquisition of the 2D PC CMR, the dogs were first hyperventilated (by increasing the respiratory rate, not the tidal volume) to reduce $\mathrm{PE}$ 'CO2 to $35 \mathrm{mmHg}(4.7 \mathrm{kPa})$. Then, expiratory apnea was induced by switching off the ventilator for the duration of the image acquisition of a single slice (5-30 seconds). This led to constantly increasing $\mathrm{PaCO} 2$ values during apnea leading to PE'CO2 of $40-50 \mathrm{mmHg}$ (5.9-6.6 $\mathrm{kPa})$ when resuming ventilation. The positive correlation of $\mathrm{CO} 2$ and stroke volume within the physiologic range (Hedenstierna and Löfström, 1973) could have an influence on stroke volume depending on the $\mathrm{PaCO} 2$ level at the moment of sequencing, meaning a rather low stroke volume when directly starting at the beginning of breath holding and a rather higher stroke volume at the end of breath holding. Since MRI sequences average multiple heartbeats over the entire length of the acquisition, this influence is minimized.

The main limitation of the study is the low number of animals. The statistical power could not be assessed in advance, since these are the first results comparing the 2D PC CMR and 4D flow CMR in dogs. The limited number of included animals has a direct effect on the quality of the available result. Based on this first information, it would be beneficial to start a clinical study including a larger number of measurements. An additional limitation was the unequal distribution of male and female dogs as well as the inclusion of a single breed. The age distribution of our population did not represent the expected population of clinical patients.

\section{Conclusion}

4D flow CMR is a rapid and feasible method to evaluate cardiac function. The established method can be replaced with the volume acquisition SENSE for the left side of the heart. On the right side, significant differences are seen and studies with a larger number of animals are needed to clarify this finding. Acceleration image acquisition with $\mathrm{k}$-t BLAST underestimates stroke in comparison to k-t SENSE and should be avoided for numeric quantification of flow parameters of examination. The main advantage using a 4D flow CMR is the independence of operator skills in placement of cardiac planes and the inclusion of the whole heart in the FOV as all imaging planes are acquired during a single acquisition reducing the time of general anesthesia of the animals. All desired measurements are postponed to post processing and performed after acquisition. This is an important point regarding clinical patients and especially patients with cardiac diseases, having a higher risk of complications during anesthesia.

The acquisition of the whole heart adding the time as the fourth dimension gives the advantage of visualization of flow plotted as streamlines (Fig. 4). The possibility of visualization of vascular flow in MRI opens the field of additional procedures and with the new technique a large amount of measurements, acquired with a single sequence, is possible.

\section{Acknowledgements}

The study was supported in part by the Albert-HeimFoundation, Bern, Switzerland.

The authors would like to thank Gérard Crelier from Gyrotools for his support.
Whole-heart 4D flow cardiac magnetic resonance in healthy dogs

N.S. Pfammatter et al. 
Whole-heart 4D flow cardiac magnetic resonance in healthy dogs

N.S. Pfammatter et al.

\section{Mesures de flux par tomographie par résonnance magnétique en $4 \mathrm{D}$ dans le cœur de chiens en bonne santé}

Lors d'examens cardiaques par résonnance magnétique (CMR), le plan pour des mesures de flux précises doit être défini perpendiculairement à la direction de flux sanguin. Dans la CMR en contraste de phases 2D (PC), le choix de ce plan se fait durant l'examen, ce qui prend du temps et peut être sujet à des problèmes. Avec la mesure de toutes les données relatives au flux sanguin dans l'ensemble du cœur au moyen d'un 4D flow CMR, on déplace le moment de ce choix dans la phase de traitement des données. La collecte d'une quantité aussi élevée de données nécessite une accélération de la technique de mesures comme par exemple SENSitivity Encoding (k-t SENSE) ou Broad-use Linear Acquisition Speed-up Technique (k-t BLAST). Le but de cette étude était de comparer la CMR 4D, accélérée avec deux méthodes différentes avec la CMR 2D bien établie, ceci sur la base de la détermination du volume d'éjection systolique au niveau des quatre valvules cardiaques. Les valeurs du volume d'éjection pour le cœur gauche (valvules aortiques et mitrales) obtenues par 4D flow CMR k-t SENSE n'étaient pas significativement différentes de celles obtenues par 2D PC CMR k-t SENSE. Par contre, des différences significatives entre les deux techniques étaient constatées au niveau des valvules pulmonaires et tricuspid. L'accélération par k-t-BLAST donnait de façon générale des valeurs du volume d'élection plus basses au niveau de toutes les valvules, à l'exception de la valvule mitrale.

\section{Misure del flusso in 4D nel cuore in cani sani con I'ausilio della risonanza magnetica cardiaca}

Al momento dell'esame cardiaco mediante risonanza magnetica (CRM) il piano per delle misure accurate del flusso deve venire definito in quanto perpendicolare alla direzione del flusso sanguigno. Già durante la CRM a contrasto di fase 2D (PC), la scelta del piano durante l'esame ha richiesto tempo ed è stata soggetta a problemi. Con la misura di tutte le informazioni del flusso sanguigno in tutto il cuore via una CRM con flusso 4D, la scelta del piano è passata ad un'elaborazione seguente. La raccolta di tali grandi quantità di dati richiede un'accelerazione della tecnica di misura quale SENSitivity Encoding (k-t SENSE) oppure Broad-use Linear Acquisition Speed-up Technique (k-t BLAST). Scopo di questo studio era di paragonare il flusso 4D della CRM, accelerato con due diversi metodi, e con la riconosciuta 2D PC CRM sulla base della determinazione dei volumi di gittata su tutte e quattro le valvole cardiache. I valori della misurazione della gittata nella parte sinistra del cuore (valvola aortica e mitrale), misurati via la CRM a flusso 4D con k-t SENSE erano significativamente differenti rispetto alle misurazioni mediante il k-t SENSE per il 2D PC CRM. Per quel che riguarda la valvola polmonare e la tricuspide si sono riscontrate differenze significative tra queste due tecniche. L'accelerazione via $\mathrm{k}-\mathrm{t}$-BLAST ha rivelato in generale valori inferiori del volume di gittata per tutte le valvole cardiache eccetto la valvola mitralica.

\section{References}

Attili A.K., Schuster A., Nagel E., Reiber J.H., van der Geest R.J.: Quantification in cardiac MRI: advances in image acquisition and processing. Int. J. Cardiovasc. Imaging. 2010, 26 Suppl 1:27-40.

Baumwart R.D., Meurs K.M., S.V. Raman.: Magnetic Resonance Imaging of Right Ventricular Morphology and Function in Boxer Dogs with Arrhythmogenic Right Ventricular Cardiomyopathy. J. Vet. Intern. Med. 2009, 23: 271-274.

Boddy K.N., Sleeper M.M., Sammarco C.D., Weisse C., Ghods S., Litt H.I.: Cardiac Magnetic Resonance in the Differentiation of Neoplastic and Nonneoplastic Pericardial Effusion. J. Vet. Intern. Med. 2011, 25: 1003-1009.

Bogaert, J., Dymarkowski, S., Taylor, A.M.: Clinical Cardiac MRI, Springer-Verlag, Berlin, 2012.

Brix L., Ringgaard S., Rasmusson A., Sorensen T.S., Kim W.Y. Three dimensional three component whole heart cardiovascular magnetic resonance velocity mapping: comparison of flow measurements from 3D and 2D acquisitions. J. Cardiovasc. Magn. Reson. 2009, 11: 3.
Carlsson M., Toger J., Kanski M., Bloch K.M., Stahlberg F., Heiberg E., Arheden H.: Quantification and visualization of cardiovascular 4D velocity mapping accelerated with parallel imaging or k-t BLAST: head to head comparison and validation at 1.5 T and 3 T. J. Cardiovasc. Magn. Reson. 2011, 13:55.

Contreras S., Vazquez J.M., Miguel A.D., Morales M., Gil F. Lopez O., Arencibia A.: Magnetic resonance angiography of the normal canine heart and associated blood vessels. Vet. J. 2008, 178:130-132.

Dennler M., Richter H., Matos J.N., Baron Toaldo M., Campagna I.U., Glaus T., Kircher P.R.: Canine cardiac MRI: Comparison of left and right ventricular stroke volume calculated based on morphology and flow. Am. J. Vet. Res. 2017, 78: $1163-1170$

Drees R., Johnson R.A., Stepien R.L., Munoz Del Rio A., François C.J.: Effects of Two Different Anesthesia Protocols on Cardiac Flow Measured by Two Dimensional Phase Contrast Magnetic Resonance Imaging. Vet. Radiol. Ultrasound 2015, 56: 168-175. 
Drees R., Johnson R.A., Stepien R.L., Munoz Del Rio A., Saunders J.H., Francois C.J.: Quantitative Planar and Volumetric Cardiac Measurements Using 64 MDCT and 3T MRI vs. Standard 2D and M-Mode Echocardiography: Does Anesthetic Protocol Matter? Vet. Radiol. Ultrasound 2015, 56: 638-657.

Dyverfeldt, P., Bissell M., Barker A.J., Bolger A.F., Carlhäll CJ., Ebbers T., Francios C.J., Frydrychowicz A., Geiger J., Giese D., Hope M.D., Kilner P.J., Kozerke S., Myerson S., Neubauer S., Wieben O., Markl M.: 4D flow cardiovascula magnetic resonance consensus statement. J. Cardiovasc. Magn. Reson. 2015, 17: 72.

Garg P., Westenberg J.J.M., van den Boogard P.J., Swoboda P.P., Aziz R., Foley J.R.J., Fent G.J., Tyl F.G.J., Coratella L., ElBaz M.S.M., van der Geest R.J., Higgins D.M., Greenwood J.P., Plein S.: Comparison of Fast Acquisition Strategies in Whole-Heart Four-Dimensional Flow Cardiac MR: TwoCenter, 1.5 Tesla, Phantom and In Vivo Validation Study. J. Magn.Reson.Imaging 2017, 17: 72

Gilbert S.H., McConnell F.J., Holden A.V., Sivananthan M.U. Dukes-McEwan J.: The potential role of MRI in veterinary clinical cardiology. Vet. J. 2010, 183: 124-134.

Hedenstierna G., Löfström J.B.: Cardiac output and venous admixture during intermittent positive pressure breathing. Influence of respiratory frequency and arterial carbon diox ide tension. Br. J. Anaesth. 1973, 45: 1201-1205.

Hockings P.D., Busza A.L., Byrne J., Patel B., Smart S.C., Reid D.G., et al.: Validation of MRI measurement of cardiac output in the dog: the effects of dobutamine and minoxidil. Toxicol. Mech. Methods. 2003, 13: 39-43.

Kim J.H., Lee M.S., Lee S.Y., Kim S.Y., Lee S.Y., Lee S.J., et al.: Contrast Echocardiography to assess left ventricular volume and function in Beagle dogs: Comparison with 3-Tesla dual source parallel cardiac magnetic resonance imaging. Vet. J. 2013; 198: 450-456.

Lee Y., Jung J., Park J., Jeong J., Jeon S., Park S., Chang J., Kang J., Lee C., Chang D.: Cardiac Magnetic Resonance Imaging of Patent Ductus Arteriosus in Three Dogs. Vet. Radiol. Ultrasound. 2017, 58: 62-75.

Lotz J., Meier C., Leppert A., Galanski M.: Cardiovascular flow measurement with phase-contrast MR imaging: basic facts and implementation. Radiographics 2002, 22: 651-671.

MacDonald K.A., Kittleson M.D., Reed T., Larson R.F., Kass P.H., Wisner E.R.: Quantification of left Ventricular Mass using Cardiac Magnetic Resonance Imaging compared with Echocardiography in domestic Cats. Vet. Radiol. Ultrasound. 2005, 46: 192-199.

MacDonald K.A., Kittleson M.D., Garcia-Nolen T., Larson R.F., Wisner E.R.: Tissue Doppler Imaging and Gradient Echo Cardiac Magnetic Resonance Imaging in Normal Cats and Cats with Hypertrophic Cardiomyopathy. J. Vet. Intern. Med. 2006, 20: 627-634.

MacDonald K.A., Kittleson M.D., Larson R.F., Kass P., Klose T., Wisner E.R.: The effect of ramipril on left ventricular mass, myocardial fibrosis, diastolic function, and plasma neurohormones in Maine Coon cats with familial hypertrophic cardiomyopathy without heart failure. J. Vet. Intern. Med. 2006, 20: 1093-1105.

Mai W., Weisse C., Sleeper M.M.: Cardiac Magnetic Resonance Imaging in normal Dogs and two Dogs with Heart Base Tumor. Vet. Radiol. Ultrasound. 2010, 51: 428-435.

Meyer J, Wefstaedt $P$, Dziallas $P$, Beyerbach $M$, Nolte I, Hungerbühler S.O.: Assessment of left ventricular volumes by use of one-, two-, and three-dimensional echocardiography versus magnetic resonance imaging in healthy dogs. Am. J. Vet. Res. 2013, 74: 1223-1230.
Sargent J., Connolly D.J., Watts V., Motskula P., Volk H.A., Lamb C.R., Luis Fuentes V.: Assessment of mitral regurgitation in dogs: comparison of results of echocardiography with magnetic resonance imaging. J. Small. Anim. Pract. 2015, 56: 641-650.

Sieslack A.K., Dziallas P., Nolte I., Wefstaedt P.: Comparative assessment of left ventricular function variables determined via cardiac computed tomography and cardiac magnetic resonance imaging in dogs. Am. J. Vet. Res. 2013, 74: 990 998.

Sieslack A.K., Dziallas P., Nolte I., Wefstaedt P., Hungerbuhler S.O.: Quantification of right ventricular volume in dogs: a comparative study between three-dimensional echocardiography and computed tomography with the reference method magnetic resonance imaging. BMC Vet. Res. 2014, 10: 242.

Stadlbauer A., van der Riet W., Globits S., Crelier G., Salomonowitz E.: Accelerated phase-contrast MR imaging: comparison of k-t BLAST with SENSE and Doppler ultrasound for velocity and flow measurements in the aorta. J. Magn. Reson. Imaging. 2009, 29: 817-824.

Stadlbauer A., van der Riet W., Crelier G., Salomonowitz E.: Accelerated time-resolved three-dimensional MR velocity mapping of blood flow patterns in the aorta using SENSE and k-t BLAST. Eur. J. Radiol. 2010, 75: 15-21.

Stankovic Z., Allen B.D., Garcia J., Jarvis K.B., Markl M.: 4D flow imaging with MRI. Cardiovasc. Diagn. Ther. 2014, 4: 173-192.

Vallee J.P., Ivancevic M.K., Nguyen D., Morel D.R., Jaconi M.: Current status of cardiac MRI in small animals. Magn. Reson. Mater. Phy. 2004, 17: 149-156.

van Ooij P., Guedon A., Marquering H.A., Schneiders J.J. Majoie C.B., van Bavel E., Nederveen A.J.: k-t BLAST and SENSE accelerated time-resolved three-dimensional phase contrast MRI in an intracranial aneurysm. Magn. Reson. Mater. Phy. 2013, 26: 261-270.

\section{Corresponding author}

Nadia S. Pfammatter

Clinic of Diagnostic Imaging

Vetsuisse Faculty Zurich

Winterthurerstrasse $258 \mathrm{c}$

$\mathrm{CH}-8057$ Zurich

Tel: +41446359126

Fax: +41 446358940

E-Mail: npfammatter@vetclinics.uzh.ch
Whole-heart 4D flow cardiac magnetic resonance in healthy dogs

N.S. Pfammatter et al.

.

.

